\title{
Fast Hardware Implementation of Sub-pixel Search Algorithm for Digital Speckle Correlation Method
}

\author{
Zejie Kuang, Guohe Zhang* and Feng Liang \\ School of electronics and Information Engineering, Xi'an Jiaotong University, Xi' an 710049 China \\ ${ }^{*}$ Corresponding author
}

\begin{abstract}
Digital speckle correlation measurement method is an important way in the areas of three-dimensional deformation and morphology measurement. It carries the advantages of non-contact, high precision and strong stability. The sub-pixel search algorithm which is used to improve the accuracy of displacement measurement is one of the key technologies in digital speckle correlation method. In order to reduce the computational complexity, the sum of absolute differences (SAD) is used as the correlation function. Special fixed-point arithmetic is designed to solve the problem of processing floating-point calculation during the hardware implementation. Experimental results show that the speed of hardware implementation is more than 600 times higher than that of software implementation. The sub-pixel search accuracy is about 0.1 pixel, and the search error is about 0.03 pixel.
\end{abstract}

Keywords-digital speckle correlation measurement method; sub-pixel search algorithm; hardware acceleration

\section{INTRODUCTION}

Digital speckle correlation measurement is an important method in the field of modern optical measurement. This method obtains the deformation and other mechanical properties by carrying out the calculation for correlativity between the two speckle fields of the tested object before and after the deformation or displacement[1]. It has the characteristics of full field non-contact, no damage, high accuracy, fast speed and high automation by matching with binocular image acquisition equipment[2-3]. Because of these characteristics, the digital speckle correlation method breaks the limitations of traditional measurement methods and creates a wide range of applications, such as: measurement of deformation of composite materials[4], detection of cancer cells by measuring the skin strain[5], measurement in a complex and severe environment[6-7], et al. The basic idea of digital speckle correlation method is to divide the search process into two parts: full-pixel search and sub-pixel search[8] Full-pixel search is performed in integer pixels which is located in a large area. Sub-pixel search is done on the basis of fullpixel, which further improves the accuracy of calculation.

Most of the current digital speckle correlation methods are based on software. Although they can get relatively fast processing speed on high performance PC, they still can not meet the needs of real-time, high-speed and high-precision. Nowadays, the demand for real-time visual processing system is becoming more and more urgent. For example, the on-line detection needs to measure the three-dimensional shape of the industrial product. This requires that the system can quickly obtain the 3D depth parallax information of images, and complete the judgment of the quality of products. It often takes tens of seconds to several minutes for computer software to process a slightly complex disparity map, which seriously affects the efficiency of detection. With the rapid development of technology of FPGA (Field Programmable Gate Array) and ASIC (Application Specific Integrated Circuit), the hardware implementation of image algorithm has been widely applied in the field of image processing. Hardware has the advantages of parallel processing mechanism and pipeline structure. The implementation of image algorithm through hardware can overcome the computational bottleneck and improve the processing speed of the system. In this paper, the gray interpolation method and the surface fitting method are analyzed experimentally, and a sub-pixel search algorithm which is suitable for hardware and meets the accuracy requirements is presented. The computation speed of the digital speckle correlation method is speed up obviously by hardware implementation and can meet the requirements of real-time high-precision measurement.

\section{The PRINCIPLE OF SUb-PIXEL SEARCH METHOD}

When using digital image processing technology to process digital images, the smallest element usually is pixels. The actual deformation or displacement of the object is often not the integer times of a pixel value, so the matching precision of the full-pixel search method cannot meet the requirements of measurement. sub-pixel can then be introduced to improve the measurement accuracy. Sub-pixel reconstruction is an effective way to improve the resolution of digital images. Sub-pixel correlation search which is based on the reconstruction of the image is one of the most important links in improving the measurement precision of the optical measurement system.

\section{A. Gray-scale Interpolation Method}

Interpolating the digital image to enlarge the resolution of the image. One of the most simple and practical interpolation methods is bilinear interpolation[9]. Although its principle is simple, it can produce satisfactory results, and it is widely used in image gray-scale interpolation algorithms. As shown in Figure I, assume that the gray-scale distribution function of the image is $\mathrm{f}(\mathrm{x}, \mathrm{y})$, and the bilinear interpolation uses the gray value $f(i, j), f(i+1, j), f(i, j+1)$ and $f(i+1, j+1)$ of the four points $(i, j),(i+1, j),(i, j+1)$ and $(i+1, j+1)$ near the best matching point $\left(x^{*}, y^{*}\right)$ to determine the gray value of the interpolation point. 
The bilinear interpolation expression is:

$$
f\left(x^{*}, y^{*}\right)=a_{00}+a_{10} x^{*}+a_{01} y^{*}+a_{11} x^{*} y^{*}
$$

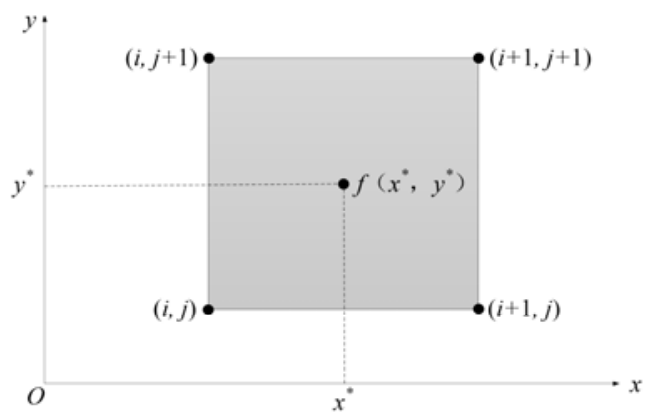

FIGURE I. THE RELATIONSHIP BETWEEN BILINEAR INTERPOLATION POINTS

Equation (1) indicates that any point $\left(x^{*}, y^{*}\right)$ within a rectangle can be determined with four known neighbors. If one of the variables in the formula is set to a constant, the function $f\left(x^{*}, y^{*}\right)$ changes linearly with respect to the other variable. That is to say, the intersection of any plane parallel to the coordinate axis and the bilinear surface is a straight line segment.

The four coefficients $a_{00}, a_{01}, a_{10}, a_{11}$ of the bilinear interpolation can be determined by the gray values of the four vertices $(i, j),(i+1, j),(i, j+1)$ and $(i+1, j+1)$ in the rectangular region. Substituting the coordinates of each vertex and the corresponding gray values into (1), the coefficients can be obtained:

$$
\left\{\begin{array}{l}
a_{00}=f(i, j) \\
a_{10}=f(i+1, j)-f(i, j) \\
a_{01}=f(i, j+1)-f(i, j) \\
a_{11}=f(i+1, j+1)-f(i+1, j)-f(i, j+1)+f(i, j)
\end{array}\right.
$$

\section{B. Surface Fitting Method}

In addition to the gray-scale interpolation method, the surface fitting method[10] is also a commonly used method in the sub-pixel solution. Through the full-pixel search, the initial value of the displacement on the speckle map can be determined. Then, the fitting model is used to fit the surface of the initial value of the displacement and the correlation coefficients of 9 pixels around it and the surface analysis function for this area is obtained. The extreme point of this analytic function is the position of the target sub-pixel. The most commonly used fitting equations at present are Gaussian surface equations and quadric surface equations. Since the fitting process of the Gaussian function requires more integer pixels, the possibility of generating a calculation error is greater. Therefore, quadric surface fitting is usually used in practical engineering applications.

As shown in Figure II, assume that the initial value of the displacement obtained by the integer pixel search method is $\left(u_{0}, v_{0}\right)$.In order to achieve a better calculation accuracy, the fitting window size is selected to be $3 \times 3$, that is, a nine-point quadratic surface fitting method. The correlation coefficient of each point near the initial value of the whole pixel displacement can be represented by the following binary quadratic function:

$$
C(x, y)=a_{0}+a_{1} x+a_{2} y+a_{3} x^{2}+a_{4} x y+a_{5} y^{2}
$$

$a_{0}, a_{1}, a_{2}, a_{3}, a_{4}$ and $a_{5}$ are the undetermined coefficients of the quadratic surface function.

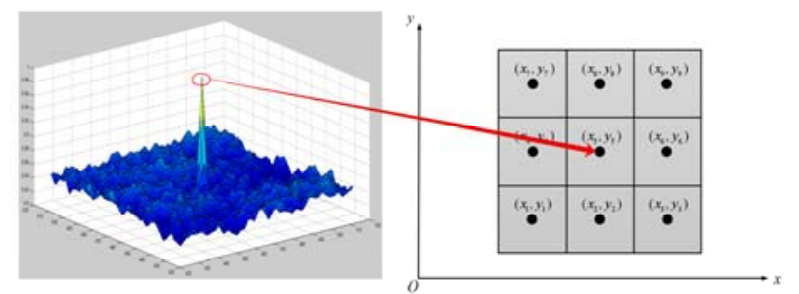

FIGURE II. SURFACE FITTING WINDOW OF THE $3 \times 3$ IMAGE

Substituting the nine point correlation coefficients in the fit window of Figure II into (3) yields the following 9 equations:

$$
\left\{\begin{array}{l}
C\left(x_{1}, y_{1}\right)=a_{0}+a_{1} x_{1}+a_{2} y_{1}+a_{3} x_{1}^{2}+a_{4} x_{1} y_{1}+a_{5} y_{1}{ }^{2} \\
C\left(x_{2}, y_{2}\right)=a_{0}+a_{1} x_{2}+a_{2} y_{2}+a_{3} x_{2}^{2}+a_{4} x_{2} y_{2}+a_{5} y_{2}^{2} \\
C\left(x_{3}, y_{3}\right)=a_{0}+a_{1} x_{3}+a_{2} y_{3}+a_{3} x_{3}^{2}+a_{4} x_{3} y_{3}+a_{5} y_{3}^{2} \\
C\left(x_{4}, y_{4}\right)=a_{0}+a_{1} x_{4}+a_{2} y_{4}+a_{3} x_{4}^{2}+a_{4} x_{4} y_{4}+a_{5} y_{4}^{2} \\
C\left(x_{5}, y_{5}\right)=a_{0}+a_{1} x_{5}+a_{2} y_{5}+a_{3} x_{5}^{2}+a_{4} x_{5} y_{5}+a_{5} y_{5}^{2} \\
C\left(x_{6}, y_{6}\right)=a_{0}+a_{1} x_{6}+a_{2} y_{6}+a_{3} x_{6}^{2}+a_{4} x_{6} y_{6}+a_{5} y_{6}{ }^{2} \\
C\left(x_{7}, y_{7}\right)=a_{0}+a_{1} x_{7}+a_{2} y_{7}+a_{3} x_{7}^{2}+a_{4} x_{7} y_{7}+a_{5} y_{7}^{2} \\
C\left(x_{8}, y_{8}\right)=a_{0}+a_{1} x_{8}+a_{2} y_{8}+a_{3} x_{8}^{2}+a_{4} x_{8} y_{8}+a_{5} y_{8}{ }^{2} \\
C\left(x_{9}, y_{9}\right)=a_{0}+a_{1} x_{9}+a_{2} y_{9}+a_{3} x_{9}^{2}+a_{4} x_{9} y_{9}+a_{5} y_{9}{ }^{2}
\end{array}\right.
$$

We can use the least square method[11] to solve the undetermined coefficients $a_{0}, a_{1}, a_{2}, a_{3}, a_{4}$, and $a_{5}$ in (4). The position of the extreme value of the fitting function $C(x, y)$ in (5) is the target sub-pixel displacement. That is, the following equation should be established:

$$
\left\{\begin{array}{l}
\frac{\partial C(x, y)}{\partial x}=a_{1}+2 a_{3} x+a_{4} y=0 \\
\frac{\partial C(x, y)}{\partial y}=a_{2}+2 a_{5} y+a_{4} x=0
\end{array}\right.
$$

From (5) we can find the position of the extreme point in the fitted surface:

$$
x=\frac{2 a_{1} a_{5}-a_{2} a_{4}}{a_{4}^{2}-4 a_{3} a_{5}}, y=\frac{2 a_{2} a_{3}-a_{1} a_{4}}{a_{4}^{2}-4 a_{3} a_{5}}
$$




\section{EXPERIMENT AND PERFORMANCE ANALYSIS}

The experiments are performed on the gray-scale interpolation and surface fitting algorithm, and comparison analysis is performed on sub-pixel search accuracy. All experiments were run on a Windows 7 32-bit operating system, an Intel CoreTM i5-2400 @ 3.10GHz CPU, and 4GB of RAM. The MABLAB R2014a software platform was used to implement the above algorithms.

\section{A. Interpolation Function Performance Analysis}

Experiments are performed on different sub-pixel interpolation functions. The experimental results show that the nearest neighbor interpolation method is simple, but the discretized surface has a high degree of dispersion, which cannot meet the accuracy requirements of this article. The bilinear interpolation method has the advantages of minor calculation and the program is easy to implement. The grayscale surface of the interpolation image is relatively smooth. After cubic spline interpolation, smooth threedimensional surface map can be achieved and the interpolation effect is better. However, the cubic spline interpolation time is several times that of bilinear interpolation. The cubic spline interpolation has high computational complexity and is not suitable for hardware implementation. Therefore, the bilinear interpolation function for sub-pixel reconstruction will be selected. The bilinear interpolation method can fully meet the requirement of positioning accuracy of 0.1 pixel.

\section{B. Comparison of Two Sub-pixel Displacement Measurement Methods}

In order to grasp the sub-pixel displacement better, the simulated speckle method was used to generate a series of simulated speckle images with known sub-pixel displacement to simulate the displacement of the object. As shown in Figure III, the pixels of the generated speckle images are $200 \times 200$, and the speckle size in each speckle image is 4 pixels, and the total number of speckles is 1500 .

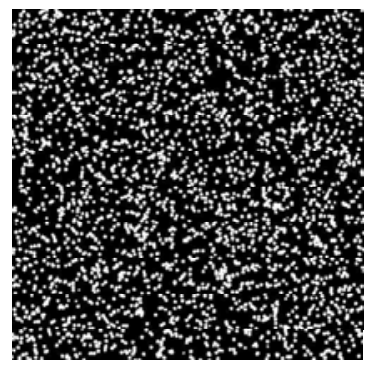

(A) REFERENCE IMAGE

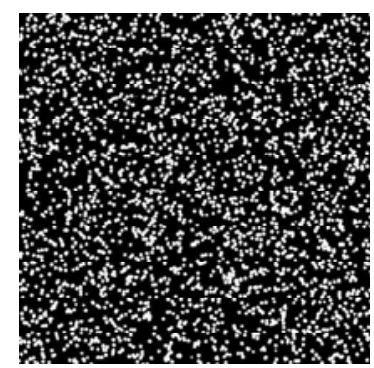

(B) TARGET IMAGE
FIGURE III. SIMULATED SPECKLE PATTERNS IN SUBPIXEL EXPERIMENTS

The target simulated speckle image remains unchanged in the vertical direction $\mathrm{v}$, and the horizontal displacement $\mathrm{u}$ is shifted by $0.05,0.1,0.15,0.2, \ldots, 0.8,0.85,0.9$, and 0.95 pixels from the reference image. Two sub-pixel search methods are used to obtain sub-pixel displacement for 50 pixels in each reference image. In order to compare the accuracy and error of the two methods better, this paper adopts error analysis theory. The error consists of the averaging error and the absolute error.
Assuming that the true displacement of the speckle image is $X_{\text {ture, }}$ the displacement of the $i$-th point is calculated as $X_{\mathrm{i}}$, $i=1,2, \ldots, 50$, and the averaging error is:

$$
\varepsilon_{\text {mean }}=X_{\text {mean }}-X_{\text {ture }}
$$

where, $X_{\text {mean }}$ is the average value calculated for N-points.

The standard error is defined as:

$$
\varepsilon_{\text {sd }}=\sqrt{\frac{1}{N-1} \sum_{i=1}^{N}\left(X_{\text {mean }}-X_{\text {ture }}\right)^{2}}
$$

The averaging error and standard error curves of the two sub-pixel search algorithms shown in Figures IV and $\mathrm{V}$ are obtained. As can be seen from Figure IV, the average error of the surface fitting is within the range of 0.07 pixel, and the average value of the gray interpolation method is within the range of 0.03 pixel. This shows that the average error of the gray interpolation method is better than the surface fitting method. As can be seen from Figure V, the standard error of the surface interpolation method is relatively large. Therefore, it can be seen from the experimental results that the accuracy of the gray-scale interpolation is higher than that of the surfacefitting method. Its error range is about 0.03 pixel and the stability is good. So, the gray-scale interpolation method is used to implement the hardware design of the sub-pixel search algorithm.

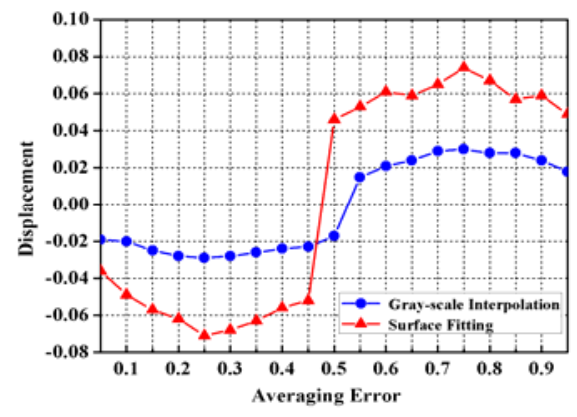

FIGURE IV. THE AVERAGING ERROR CURVE

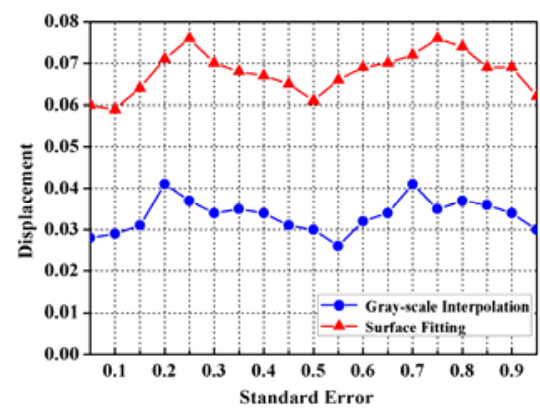

FIGURE V. THE STANDARD ERROR CURVE 


\section{HARDWARE CIRCUIT DESIGN AND IMPLEMENTATION}

Sub-pixel search adopts gray-scale interpolation method and bilinear interpolation function is used for sub-pixel interpolation. When the full-pixel search determines the best matching point, we believe that the true matching point exists within the eight-neighborhood range of the initial pixel. Therefore, we need to interpolate $3 \times 3$ pixel points in the neighborhood of the initial value of displacement. In order to achieve the accuracy requirement of 0.1 pixel, it is necessary to insert 100 sub-pixel points in the $3 \times 3$ region and perform subpixel reconstruction with an accuracy of 0.02 pixel. Then a subpixel search is performed in a $100 \times 100$ search region to determine the sub-pixel displacements, resulting in a more accurate optimal matching point. In order to reduce the computational complexity of sub-pixel search, this paper uses the sum of absolute differences (SAD) algorithm to calculate the correlation coefficients of sub-pixel search. The hardware design of sub-pixel algorithm mainly includes bilinear interpolation calculation unit and related search calculation unit.

The pipeline structure is used to design the hardware circuit of the interpolation arithmetic unit. In the hardware structure of the related search calculation unit, the data obtained by the subpixel interpolation is written into the DPRAM cache unit for parallel processing. A 25-bit integer is used to represent fixedpoint decimals. The most significant bit represents the sign bit, and the middle 8 bits represent integer bits, and the lower 16 bits represent decimal bits. We use the sum of absolute differences as sub-pixel correlation coefficient for correlation calculation. First, the absolute value of the difference between data of the reference sub-area and the search sub-area is calculated, and data are sent to the register groups. Then subtractor array calculates the absolute value of the difference between the two sub-area. Finally, the pipeline adder calculates the sum of absolute differences and finds the position corresponding to its maximum value.

Mentor Graphics Modelsim SE-64 10.2c is used to perform functional simulation of the RTL code which has been verified by FPGA. The frequency is $100 \mathrm{MHz}$. The input specklegrams use the simulated speckle method to generate several groups of speckle images with a size of $512 \times 512$. In the simulation process, the results of sub-pixel matching are output through a file, and the recorded results are compared with the results of the MATLAB test. The comparison shows that the simulation results of Modelsim are consistent with those of MATLAB. The hardware simulation time is compared with the software. The results are shown in Table I:

TABLE I. COMPARISON OF SIMULATION TIME BETWEEN HARDWARE AND SOFTWARE

\begin{tabular}{|c|c|c|c|c|c|c|}
\hline & \multirow[b]{2}{*}{$\begin{array}{c}\text { Experiment } \\
\text { platform }\end{array}$} & \multirow[b]{2}{*}{$\begin{array}{c}\text { Searc } \\
\text { hing } \\
\text { area }\end{array}$} & \multirow[b]{2}{*}{$\begin{array}{l}\text { Temp } \\
\text { late } \\
\text { size }\end{array}$} & \multicolumn{3}{|c|}{ Matching time } \\
\hline & & & & $\begin{array}{c}1- \\
\text { poin } \\
t\end{array}$ & $\begin{array}{c}64- \\
\text { point } \\
\text { s }\end{array}$ & $\begin{array}{c}100- \\
\text { point } \\
\text { s }\end{array}$ \\
\hline $\begin{array}{c}\text { Hardwar } \\
\mathrm{e}\end{array}$ & $\begin{array}{c}\text { Intel-i5 } 2400 \\
3.1 \mathrm{GHz} \\
\end{array}$ & \multirow{2}{*}{$\begin{array}{c}256 \\
\times \\
256\end{array}$} & \multirow{2}{*}{$\begin{array}{c}31 \\
\times \\
31\end{array}$} & $\begin{array}{c}1.36 \\
\mathrm{~s}\end{array}$ & $\begin{array}{c}14.8 \\
7 \mathrm{~s} \\
\end{array}$ & $\begin{array}{c}22.7 \\
6 \mathrm{~s} \\
\end{array}$ \\
\hline $\begin{array}{c}\text { Softwar } \\
\text { e }\end{array}$ & $\begin{array}{c}\text { Modelsim } 10 . \\
\text { 2c } \\
\text { (a) } 100 \mathrm{MHz}\end{array}$ & & & $\begin{array}{c}0.52 \\
\mathrm{~ms}\end{array}$ & $\begin{array}{l}23.0 \\
9 \mathrm{~ms}\end{array}$ & $\begin{array}{l}37.6 \\
2 \mathrm{~ms}\end{array}$ \\
\hline
\end{tabular}

The results show that the speed of hardware design proposed in this paper is about 2,000 times faster than software when performing single-point matching. The speed of hardware is 600 times faster than the software when performing multipoint matching. It only takes 520 microseconds when the hardware performs a single-point match. It's only a few tens of milliseconds when doing a 100-point match. Therefore, the hardware design of this article achieves the effect of real-time processing. In terms of accuracy, the design of this paper can reach 0.1 pixel, and the fluctuation range of error is kept at 0.03 pixel.

\section{CONCLUSION}

In this paper, two sub-pixel search algorithms have been tested for accuracy. Experiments show that the gray-scale interpolation method has a search accuracy of 0.1 pixel and the error fluctuation range is 0.03 pixel. It is suitable for hardware implementation and meets accuracy requirements. The sum of absolute differences (SAD) in the matching algorithm is used as the correlation function of the sub-pixel search. It reduces the computational complexity of sub-pixel search. And the problem that hardware is difficult to process floating-point calculation is effectively solved by fixed-point arithmetic. Combined with the characteristics of hardware of parallel structure and pipeline technology, the hardware circuit of subpixel search algorithm is implemented. Through simulation, the design function and performance are verified, and the operating speed of hardware is compared with the software. The results show that the function of hardware design is correct. Under the premise of no loss of precision, the operating speed of hardware is more than 600 times faster than software.

\section{ACKNOWLEDGMENT}

This work was supported in part by the National Natural Science Foundation of China under Grant 61474093.

\section{REFERENCES}

[1] DONG H, ZHOU Y, GUO J, ZHANG W Y, WANG W S. Digital Speckle Pattern Interferometry for Deformation Measurement[J]. Acta Photonica Sinica, 2010, 39(s1): 19-22.

[2] CHEN Z X, LIANG J, GUO C. Application of digital speckle correlation method to deformation measurement $[\mathrm{J}]$. Optics and Precision Engineering, 2011, 19(7): 1480-1485.

[3] YU G, WANG S G, YU J H. Technology of digital speckle pattern interferometry and its applications[J]. Laser Technology, 2002, 26(3): 237-240.

[4] SUN T, LIANG J, CAI Y, WANG Y Q. Measurement of deformations for copper/aluminum clad under tension with digital speckle correlation[J]. Optics and Precision Engineering, 2012, 20(12): 2599-2606.

[5] Krehbiel J D, Lambros J, Viator J A, NR Sottos. Digital Image Correlation for Improved Detection of Basal Cell Carcinoma[J]. Experimental Mechanics, 2010, 50(6): 813-824.

[6] Lyons J S, Liu J, Sutton M A. High-temperature deformation measurements using digital-image correlation[J]. Experimental Mechanics, 1996, 36(1): 64-70.

[7] Anwander M, Zagar B G, Weiss B, H Weiss. Noncontacting strain measurements at high temperatures by the digital laser speckle technique[J]. Experimental Mechanics, 2000, 40(1): 98-105. 
[8] Pan B, Qian K, Xie H, A Asundi. Two-dimensional digital image correlation for in-plane displacement and strain measurement: a review[J]. Measurement Science \& Technology, 2009, 20(6): 152-154.

[9] SUN Y L, LI S X, LI J Z. Investigation and modification of the digital speckle correlation method[J]. Acta Photonica Sinica, 2001, 30(1): $54-57$.

[10] LI X Z, LIU H H, ZHEN Z Q, LI L B, CHEN Q D. Investigation of Curved Surface Fitting Method in Sub-pixel Displacement Digital Speckle Correlation Measurement[J]. Laser \& Optoelectronics Progress, 2009, 46(8): 72-75.

[11] Benckert L R, Sjödahl M. Electronic speckle photography: analysis of an algorithm giving the displacement with subpixel accuracy[J]. Applied Optics, 1993, 32(13):2278-84. 